Article

\title{
Platelet-Rich Fibrin Can Neutralize Hydrogen Peroxide-Induced Cell Death in Gingival Fibroblasts
}

\author{
Zahra Kargarpour ${ }^{1}$, Jila Nasirzade ${ }^{1}$, Francesca Di Summa ${ }^{1}$, Layla Panahipour ${ }^{1}{ }^{(1)}$, \\ Richard J. Miron ${ }^{2}$ and Reinhard Gruber 1,2,* \\ 1 Department of Oral Biology, Medical University of Vienna, 1090 Vienna, Austria; \\ zahra.kargarpooresfahani@meduniwien.ac.at (Z.K.); jila.nasirzaderajiri@meduniwien.ac.at (J.N.); \\ francesca.disumma@studenti.unipr.it (F.D.S.); layla.panahipour@meduniwien.ac.at (L.P.) \\ 2 Department of Periodontology, School of Dental Medicine, University of Bern, 3012 Bern, Switzerland; \\ richard.miron@zmk.unibe.ch \\ * Correspondence: reinhard.gruber@meduniwien.ac.at; Tel.: +43-140-070-2660
}

Received: 5 June 2020; Accepted: 23 June 2020; Published: 26 June 2020

Abstract: Hydrogen peroxide is a damage signal at sites of chronic inflammation. The question arises whether platelet-rich fibrin (PRF), platelet-poor plasma (PPP), and the buffy coat can neutralize hydrogen peroxide toxicity and thereby counteract local oxidative stress. In the present study, gingival fibroblasts cells were exposed to hydrogen peroxide with and without lysates obtained from PRF membranes, PPP, heated PPP $\left(75^{\circ} \mathrm{C}\right.$ for $\left.10 \mathrm{~min}\right)$, and the buffy coat. Cell viability was examined by trypan blue staining, live-dead staining, and formazan crystal formation. Cell apoptosis was assessed by cleaved caspase-3 Western blot analysis. Reverse transcription-quantitative polymerase chain reaction (RT-PCR) was utilized to determine the impact of PRF lysates on the expression of catalase in fibroblasts. It was reported that lysates from PRF, PPP, and the buffy coat-but not heated PPP-abolished the hydrogen peroxide-induced toxicity in gingival fibroblasts. Necrosis was confirmed by a loss of membrane integrity and apoptosis was ruled out by the lack of cleavage of caspase-3. Aminotriazole, an inhibitor of catalase, reduced the cytoprotective activity of PRF lysates yet blocking of glutathione peroxidase by mercaptosuccinate did not show the same effect. PRF lysates had no impact on the expression of catalase in gingival fibroblasts. These findings suggest that PRF, PPP, and the buffy coat can neutralize hydrogen peroxide through the release of heat-sensitive catalase.

Keywords: platelet-rich-fibrin; platelets; catalase; oxidative stress; acute toxicity; apoptosis; ulcer; wound healing; dentistry

\section{Introduction}

Platelet-rich fibrin (PRF) is a fibrin-rich network that serves as a scaffold for bioactive molecules released from platelets being widely used in regenerative dentistry [1]. PRF has been shown to limit dimensional changes of the alveolar ridge post-extraction [2]. Furthermore, PRF has been utilized as an adjuvant for peri-implantitis therapy [3-5]. In general medicine, PRF has also been utilized to promote the healing and wound closure of chronic ulcers [6] and resurfacing of full-thickness burns [7]. The beneficial effects can be explained by the PRF intrinsic growth factors triggering cell responses of PRF including its ability to promote cell proliferation, migration, and differentiation [8,9]. Apart from its antimicrobial activity [10], PRF can also suppress inflammation [11] and osteoclastogenesis [12]. The complex effects of PRF presumably exceed those mediated simply by growth factors and we, therefore, proposed here that PRF may also support the local tissues by counteracting the reactive oxygen species and thereby minimizing oxidative stress. 
Reactive oxygen species (ROS) are highly reactive molecules generated by local organisms and include hydrogen peroxide $\left(\mathrm{H}_{2} \mathrm{O}_{2}\right)$, superoxide, and hydroxyl radical [13]. Under physiological conditions, hydrogen peroxide plays a pivotal role in cell growth and proliferation. Overproduction of hydrogen peroxide causes oxidative stress that may also lead to cellular damage and apoptotic cell death [14]. Hydrogen peroxide is neutralized by catalase (EC 1.11.1.6) and glutathione peroxidase (GPX; EC 1.11.1.9). Activated platelets liberate both catalase and glutathione peroxidase [15-17]. Also, erythrocytes accumulating next to the buffy coat layer at the transition of yellow and the red clot, are a rich source of catalase and glutathione peroxidase [18]. Catalase efficiently converts hydrogen peroxide into water and molecular oxygen. Glutathione peroxidase removes hydrogen peroxide at the expense of glutathione and requires selenium. Since platelets can consume hydrogen peroxide produced by neutrophils [19], it is plausible that platelets neutralize hydrogen peroxide and thereby protect cells involved in granulation tissue formation.

Recently, we have shown that supernatants of activated platelets efficiently neutralize toxic concentrations of hydrogen peroxide [20]. This neutralization, however, did not occur in the presence of the catalase inhibitor aminotriazole and was independent of glutathione peroxidase [17]. Similar findings were observed with erythrocytes. Inhibition of erythrocyte catalase abrogates their protective effect towards neutralizing high levels of exogenous hydrogen peroxide [18]. It is thus conceivable that PRF contains catalase. Catalase, however, is a heat-sensitive protein suggesting that pasteurization of platelet products may lose their activity [21]. This aspect is relevant as heating of liquid platelet-poor plasma (PPP) is applied to prolong the resorption of albumin gels $[22,23]$ also when preparing injectable PRF consisting of autologous albumin gel and a liquid buffy coat (Alb-PRF) [24]. The aim of this study was, therefore, to examine whether standard PRF, standard and heated PPP, as well as the buffy coat layer, could neutralize the toxic concentration of exogenous hydrogen peroxide.

\section{Materials and Methods}

\subsection{Cell Culture}

Human gingiva was harvested during the extraction of impacted wisdom teeth from young and healthy patients who stayed anonymous and had given informed and written consent. A total of three strains of fibroblasts were established by explant cultures. Cells growing out from the gingiva explants were further expanded and stored in liquid nitrogen at low passage. Fibroblasts expanded for fewer than 10 passages were used for the experiments. Approval was obtained from the Ethics Committee of the Medical University of Vienna (EK NR 631/2007). A total of three strains of fibroblasts were established by explant cultures and fewer than 10 passages were used for the experiments. Gingival fibroblasts were grown and supplemented with 1\% antibiotics (Sigma Aldrich, St. Louis, MO, USA) and 10\% fetal calf serum (Bio\&Sell GmbH, Nuremberg, Germany). The cells were exposed to the respective treatments for another $24 \mathrm{~h}$ under standard conditions at $37{ }^{\circ} \mathrm{C}, 5 \% \mathrm{CO}_{2}$ and $95 \%$ humidity.

\subsection{Preparation of Platelet-Rich Fibrin}

PRF membranes were prepared after the approval of the ethics committee of the Medical University of Vienna (1644/2018) and volunteers signed informed consent. All experiments were performed in accordance with relevant guidelines and regulations. Venous blood was collected at the University Clinic of Dentistry from six non-fasting healthy volunteers (21G, Greiner Bio-One, Kremsmünster, Austria), each donating six plastic spray-coated silica tubes (BD Vacutainer ${ }^{\circledR}$ Plymouth, UK) supporting spontaneous blood coagulation. PRF clots were produced by centrifugation at $400 \mathrm{~g}$ for $12 \mathrm{~min}$ utilizing a centrifuge device (Z 306 Hermle Universal Centrifuge, Wehingen, Germany) with universal swing-out rotors (146 $\mathrm{mm}$ at the max). The yellow PRF clot was separated from the remaining red clot and compressed between two layers of sterile gauzes to generate PRF membranes. PRF membranes were transferred into serum-free medium ( $1 \mathrm{~cm}$ PRF membrane $/ \mathrm{mL}$ ) and subjected to repeated freeze-thawing and sonication (Sonopuls 2000.2, Bandelin electronic, Berlin, Germany). After centrifugation at 15,000 g 
for $10 \mathrm{~min}$ (Eppendorf AG, Hamburg, Germany), the lysates were subjected to sterile filtration and stored at $-20^{\circ} \mathrm{C}$ prior to the analysis [11]. In indicated experiments, PRF membranes were transferred into a serum-free medium and the conditioned medium harvested after 24 and $72 \mathrm{~h}$.

\subsection{Preparation of Blood Fractions}

For the preparation of albumin gels [24], venous blood was collected (21 G, Greiner Bio-One, Kremsmünster, Austria) in plastic tubes ("No Additive", Greiner Bio-One GmbH, Kremsmünster, Austria) and centrifuged at $700 \mathrm{~g}$ for $8 \mathrm{~min}$. The approximately $4 \mathrm{~mL}$ PPP, the $1 \mathrm{~mL}$ buffy coat layer, and the adjacent erythrocyte fraction were collected. To generate albumin gels (Alb-gel), PPP was heated at $75{ }^{\circ} \mathrm{C}$ for $10 \mathrm{~min}$ (Eppendorf, Thermomixer F1.5, Hamburg, Germany) and placed on crushed ice thereafter [25]. In another approach, $1 \mathrm{~mL}$ samples were pipetted precisely from the upper layer downward to consequently end up with 10 fractions. Each preparation was subjected to repeated freeze-thawing, transferred into an equal volume of serum-free medium and sonicated. After centrifugation at 15,000 $\mathrm{g}$ for $10 \mathrm{~min}$, the lysates were subjected to sterile filtration and stored at $-20{ }^{\circ} \mathrm{C}$ prior to the analysis.

\subsection{Cell Viability Assay}

For the viability assay, the various lysates were mixed with $3 \mathrm{mM} \mathrm{H}_{2} \mathrm{O}_{2}$ (Sigma Aldrich, St. Louis, $\mathrm{MO}, \mathrm{USA}$ ) and incubated for $10 \mathrm{~min}$ at room temperature. Catalase and glutathione peroxidase activities in PRF lysates were blocked by incubation with $100 \mathrm{mM}$ aminotriazole (Sigma Aldrich, St. Louis, MO, USA) and 10 mM mercaptosuccinate (Sigma Aldrich, St. Louis, MO, USA), respectively. The cells were exposed to $10 \%$ of PRF lysates. After three hours of exposure, a cell viability assay was performed. For cell viability, MTT solution (Sigma Aldrich, St. Louis, MO, USA) at a final concentration of $0.5 \mathrm{mg} / \mathrm{mL}$ was added to each well of a microtiter plate and incubated for $2 \mathrm{~h}$ at $37^{\circ} \mathrm{C}$, $5 \% \mathrm{CO}_{2}$ and $95 \%$ humidity. The medium was removed and the formazan crystals were solubilized with dimethyl sulphoxide (Sigma Aldrich, St. Louis, MO, USA). The optical density was measured at $570 \mathrm{~nm}$. The data from independent experiments are presented as percentages of the optical density in the treatment groups normalized to the unstimulated control that was considered $100 \%$ viability regardless of the optical density.

\subsection{Trypan Blue Staining and Live-Dead Staining}

The lysates were incubated with $3 \mathrm{mM} \mathrm{H}_{2} \mathrm{O}_{2}$ for 10 min prior to cell stimulation for three hours. For testing the cellular membrane integrity, 0.4\% trypan blue (Sigma Aldrich, St. Louis, MO, USA) diluted in PBS was added to each well and incubated for $10 \mathrm{~min}$ at room temperature. Trypan blue was discarded and the cells were examined by light microscopy. Cell viability was further confirmed using Live-dead staining assay kit according to the instructions of the manufacturer (Enzo Life Sciences, Inc., Lausanne, Switzerland).

\subsection{Visualizing Bubble Assay and Bubble Microscopic Screening}

Catalase rapidly converts hydrogen peroxide into water and molecular oxygen, and it is the oxygen that can be visualized using a bubble assay [26]. In brief, a solution containing $15 \%$ hydrogen peroxide and $0.5 \%$ Triton X-100 (Sigma Aldrich, St. Louis, MO, USA) was mixed with the equal volume of the various fractions or standard concentrations of bovine catalase in transparent round-bottomed test tubes (VWR International). The catalase-dependent production of oxygen was represented by the height of the foam generated. The experiments were carried out in triplicate. For rapid bubble screening, $100 \mu \mathrm{L}$ of lysates were exposed to $10 \mu \mathrm{L}$ of $\mathrm{H}_{2} \mathrm{O}_{2}$ on a glass slide and the reaction was recorded on the microscope by video and photograph. 


\subsection{Western Blot Analysis}

Gingival fibroblasts were serum-starved overnight followed by three hours of stimulation with $100 \mu \mathrm{M}$ etoposide, a cell-permeable inhibitor of topoisomerase II inhibitor (Calbiochem, Merck KGaA, Darmstadt, Germany), or $3 \mathrm{mM} \mathrm{H}_{2} \mathrm{O}_{2}$. Cell extracts containing protease inhibitors (Sigma Aldrich, St. Louis, MO, USA) were separated by SDS-polyacrylamide gel electrophoresis and transferred onto polyvinylidene difluoride membranes (Roche Diagnostic, Mannheim, Germany). The membranes were blocked with 5\% dry milk and binding of the primary antibody cleaved caspase-3 (Cell Signaling Technology, Danvers, MA, USA) was detected with an appropriate secondary antibody directly labeled with peroxidase. Chemiluminescence signals were visualized with the ChemiDoc imaging system (Bio-Rad Laboratories, Inc. CA, USA).

\subsection{Reverse Transcription Quantitative Polymerase Chain Reaction}

Following stimulating the gingival fibroblast cells with $200 \mu \mathrm{M}$ etoposide and $3 \mathrm{mM}$ $\mathrm{H}_{2} \mathrm{O}_{2}$ for three hours, total RNA was isolated (ExtractMe, Blirt S.A., Gdańsk, Poland) and reverse transcription (RT) was performed (LabQ, Vienna, Austria). RT-PCR was done using the manufacturer's instructions (LabQ, Vienna, Austria). Primer sequences were hBCL2-F: AGTACCTGAACCGGCACCT, hBCL2-R: GCCGTACAGTTCCACAAAGG; hGAPDH-F: AAGCCACATCGCTCAGACAC, hGAPDH-R: GCCCAATACGACCAAATCC. Relative gene expression was calculated with the delta-delta CT method using a software (CFX Maestro TM, BioRad, Hercules, CA, USA). Reactions were run in duplicates (Bio-Rad Laboratories, Inc. CA, USA).

\subsection{Statistical Analysis}

All experiments were performed three to five times. Bars show the mean and standard deviation of the cumulative data from the means of independent experiments. Statistical analysis was based on Mann-Whitney U test and Kruskal-Wallis test with Dunn multiple comparisons correction. The analysis was performed using statistical software. Significance was set at $p<0.05$.

\section{Results}

\subsection{PRF Lysates Rescue Cells from Acute Hydrogen Peroxide Toxicity}

First, we analyzed the effects of PRF lysates on human gingival fibroblasts treated with or without hydrogen peroxide. Hydrogen peroxide led to a rapid disruption of the cell membranes indicated by the uptake of trypan blue which was not observed in the presence of PRF lysates (Figure 1A). Similarly, PRF lysates inhibited the acute toxicity of hydrogen peroxide indicated by live-dead staining (Figure 1A). In support of this observation, PRF lysates prevented hydrogen peroxide-induced reduction of formazan crystals (Figure 1B). Together, these results suggest that PRF lysates attenuate hydrogen peroxide-induced acute necrotic cell death.
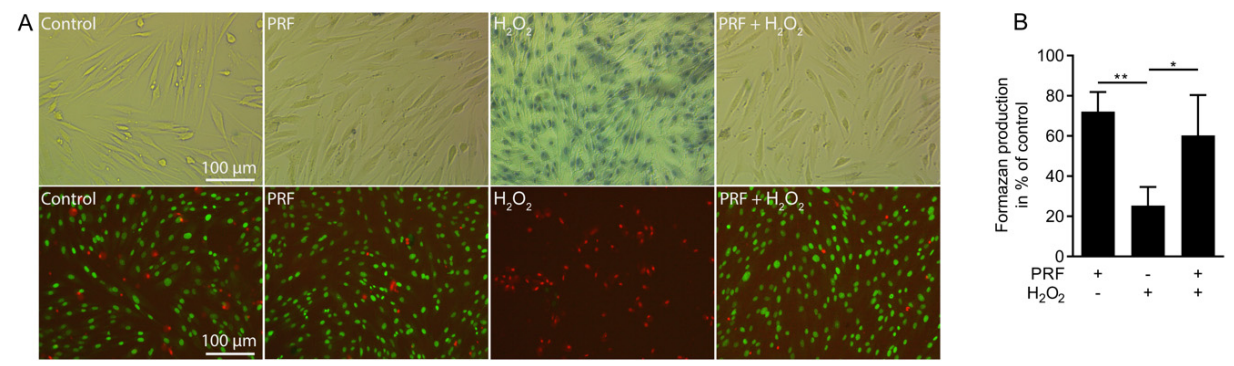

Figure 1. Platelet-rich fibrin (PRF) lysates rescue cells from acute hydrogen peroxide toxicity. Gingival fibroblasts (A) were exposed to $10 \%$ PRF lysates in the presence or absence of $3 \mathrm{mM}$ $\mathrm{H}_{2} \mathrm{O}_{2}$ for $3 \mathrm{~h}$. 
Necrotic cell death is shown by the uptake of trypan blue that however was not observed in the presence of PRF lysate. Live-dead staining was performed in gingival fibroblasts with viable cells appearing in green and dead cells in red. (B) Cell viability is represented by formazan production indicated in the percentage of unstimulated controls. The results from these experiments demonstrate that stimulation with $10 \%$ PRF lysates could neutralize the necrotic cell death caused by $\mathrm{H}_{2} \mathrm{O}_{2}$. Data represent the mean \pm SD relative to the control. $n=4 .{ }^{*} p<0.05,{ }^{* *} p<0.01$, by two-tailed Mann-Whitney test.

\subsection{Hydrogen Peroxide Toxicity Occurs by Necrosis but Not by Apoptosis}

To rule out the involvement of apoptosis, we utilized etoposide, a cell-permeable inhibitor of topoisomerase II inhibitor, to provoke apoptosis and cleavage of caspase- 3 in gingival fibroblasts. Gene expression analysis showed that the level of the pro-apoptotic marker gene B cell lymphoma-2 (BCL2) increased in the presence of etoposide, but not with hydrogen peroxide (Figure 2A). This finding is further supported by Western blot analysis based on the endogenous levels of the large fragment $(17 / 19 \mathrm{kDa})$ of activated caspase-3. We report here that etoposide, but not $3 \mathrm{mM}$ hydrogen peroxide, increased the cleavage of caspase-3 into the two fragments (Figure 2B). These findings confirm that hydrogen peroxide-induced cell death is not mediated by apoptosis. These observations suggest that the sharp decrease in cell viability is a consequence of necrosis rather than apoptosis.

A

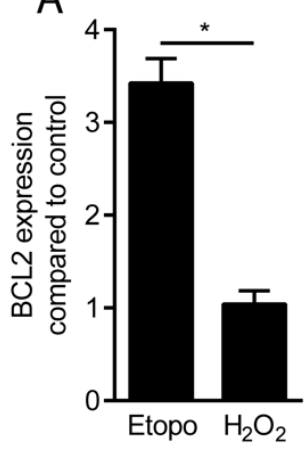

B

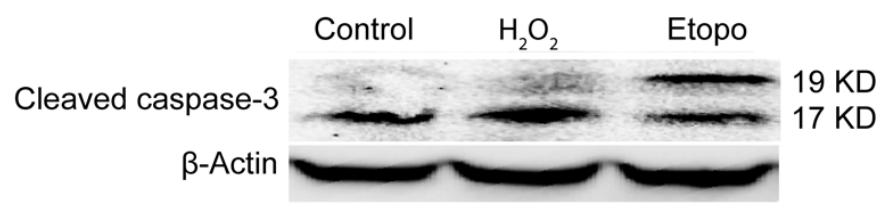

Figure 2. Hydrogen peroxide toxicity occurs by necrosis but not by apoptosis. Apoptosis of gingival fibroblasts was assessed by (A) evaluating the expression of the BCL2 gene and with (B) Western blot analysis of cleaved caspase- 3 treated with $\mathrm{H}_{2} \mathrm{O}_{2}$ at $3 \mathrm{mM}$ and etoposide at $200 \mu \mathrm{M}$ for $3 \mathrm{~h}$. It is indicated that $\mathrm{H}_{2} \mathrm{O}_{2}$ was not able to induce cleavage of caspase- 3 while it was cleaved by apoptosis inducer, etoposide. All together it suggests that the decrease in cell viability is a consequence of necrosis rather than apoptosis (Etopo: Etoposide). $n=3$. Data represent the mean \pm SD. ${ }^{*} p<0.05$, by two-tailed Mann-Whitney test.

\subsection{PRF Conditioned Medium Protects Cells from Necrotic Cell Death}

Next, we investigated whether PRF conditioned medium could neutralize the hydrogen peroxide toxicity. PRF conditioned medium obtained after $24 \mathrm{~h}$ protected gingival fibroblasts from the acute toxicity of hydrogen peroxide indicated by the uptake of trypan blue and live-dead staining (Figure 3A), and the formation of formazan crystals (Figure 3B). PRF conditioned medium obtained after $72 \mathrm{~h}$ however, failed to protect the cells from cell death. These findings indicate that conditioned medium harvested from fresh PRF membranes can suppress hydrogen peroxide-induced cell death. 

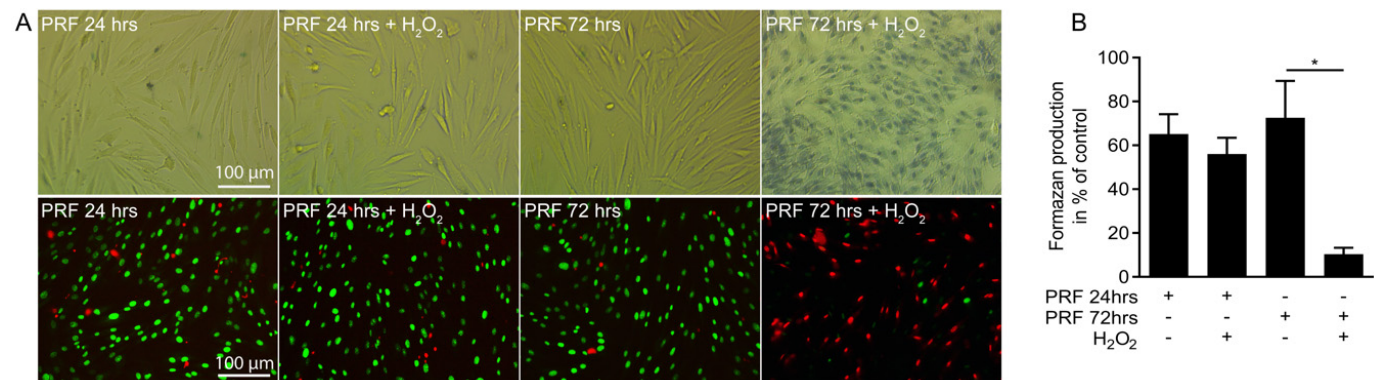

Figure 3. PRF conditioned medium protects cells from necrotic cell death. Gingival fibroblasts (A) were exposed to $10 \%$ PRF conditioned medium collected at 24 and 72 hours in the presence or absence of $3 \mathrm{mM} \mathrm{H}_{2} \mathrm{O}_{2}$ for 3 hours. Necrotic cell death is shown by the uptake of trypan blue. Live-dead staining was performed in gingival fibroblasts with viable cells appearing in green and dead cells in red. (B) Cell viability is represented by formazan production indicated in percentage of unstimulated controls. The results from these experiments demonstrated that stimulation with $10 \%$ PRF conditioned media obtained at 24 hours preserves the cells from necrosis induced by $\mathrm{H}_{2} \mathrm{O}_{2}$. Data represent the mean \pm SD relative to the control. $n=4$. Data represent the mean \pm SD. ${ }^{*} p<0.05$, by two-tailed Mann-Whitney test.

\subsection{Blocking Catalase Attenuates the Activity of PRF Lysate in Preventing the Cells from Cell Death}

To examine whether the protective activity of PRF lysate involved catalase, the catalase inhibitor aminotriazole was used. Aminotriazole $(100 \mathrm{mM})$ abolished the cytoprotective activity of PRF lysates (Figure 4A). Similar to purified platelets [17], blocking of glutathione peroxidase by mercaptosuccinate failed to reduce the cytoprotective activity of PRF lysates (data not shown). Considering that catalase is heat-sensitive [26], PRF lysates heated at $95^{\circ} \mathrm{C}$ for $30 \mathrm{~min}$ lost the ability to neutralize hydrogen peroxide (Figure 4B). Based on a standard curve, undiluted PRF lysates contain approximately $30 \mathrm{unit} / \mathrm{mg}$ of catalase (Supplementary Figure S1). Taken together, these data suggest that the protective activity of PRF lysates is mediated by catalase.
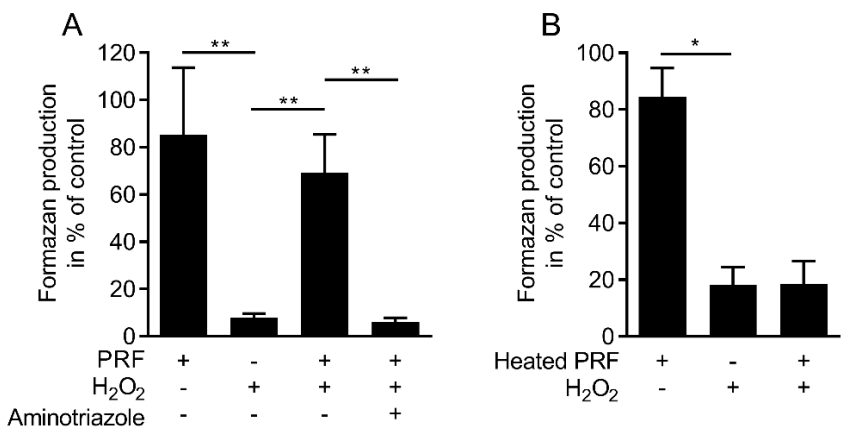

Figure 4. Blocking catalase attenuates the activity of PRF lysate in preventing cell death. Gingival fibroblasts (A) were exposed to $10 \%$ PRF in the presence or absence of $3 \mathrm{mM} \mathrm{H}_{2} \mathrm{O}_{2}$ and $100 \mathrm{mM}$ catalase inhibitor, aminotriazole. Cell viability is measured by the conversion of MTT into formazan crystals. (B) MTT assay also demonstrated that heated PRF is not able to neutralize $\mathrm{H}_{2} \mathrm{O}_{2}$. $n=3-5$. Data represent the mean \pm SD. ${ }^{*} p<0.05,{ }^{* *} p<0.01$, by two-tailed Mann-Whitney test.

\subsection{PRF Capacity in Neutralizing Hydrogen Peroxide Is Limited}

To figure out the concentration where PRF lost the ability to neutralize hydrogen peroxide, a dose-response experiment was carried out. Gingival fibroblasts were exposed to different concentrations of hydrogen peroxide in the presence or absence of $10 \%$ PRF lysates. The formazan production indicated that 10,30 , and $100 \mathrm{mM}$ hydrogen peroxide, but not $300 \mathrm{mM}$ were neutralized by $10 \%$ PRF (Figure 5A). To measure the stability of catalase, PRF lysates were stored at $37^{\circ} \mathrm{C}$ for 24,48 , and $72 \mathrm{~h}$. The results demonstrated that catalase in PRF lysates kept its activity over time (Figure 5B). 
Thus, PRF lysates could effectively neutralize high amounts of hydrogen peroxide and maintain the activity upon storage.
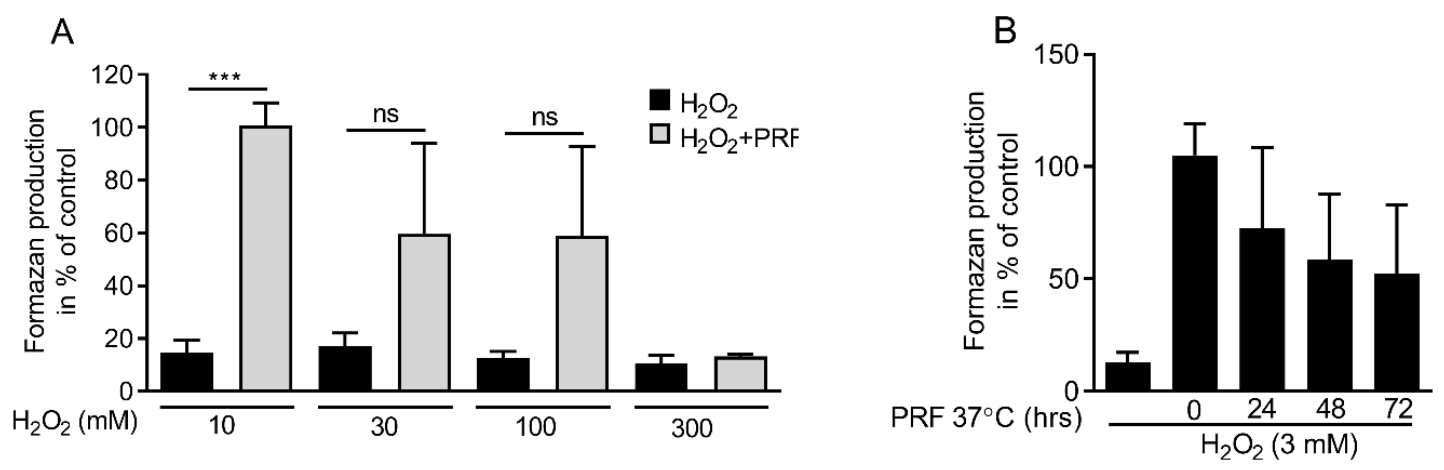

Figure 5. PRF capacity in neutralizing hydrogen peroxide is dose-dependent. (A) Gingival fibroblast cells were exposed to PRF lysates in the presence of various doses of $\mathrm{H}_{2} \mathrm{O}_{2}(10,30,100,300 \mathrm{mM})$. MTT results confirmed cell viability in the presence of PRF lysates for all the groups except $300 \mathrm{mM}$. (B) The neutralizing capacity of PRF lysates incubated at $37^{\circ} \mathrm{C}$ for 24,48 , and $72 \mathrm{~h}$ were also tested in the presence of $3 \mathrm{mM} \mathrm{H}_{2} \mathrm{O}_{2}$. The results suggest that catalase activity was decreased in a time-dependent manner. $n=3-5$. Data represent the mean \pm SD. ${ }^{* * *} p<0.001$ and ns $=$ not significant, by two-tailed Mann-Whitney test.

\subsection{Regular PPP, Buffy Coat and Red Blood Clot but Not Albumin Gel Neutralize Hydrogen Peroxide}

The next experiments were based on the heat sensitivity of catalase [27] based on the recent introduction of an injectable PRF mixture where the PPP is heated at $75^{\circ} \mathrm{C}$ for $10 \mathrm{~min}$ to create denatured albumin (albumin gel) [24]. Viability assays confirmed that lysates of heated PPP lose the catalase activity, while lysates from regular PPP, the buffy coat layer and the remaining red clot neutralize hydrogen peroxide. These data support the assumption that the catalase activity of the injectable PRF mixture is present in the PPP and buffy coat layer but not the albumin gel due to heating (Figure 6). The findings were further confirmed by visualizing and quantifying bubble assay and microscopic bubble screening (Supplementary Figures S2-S3 and Table S1). In this assay, the buffy coat layer and the remaining red clot fraction cause significantly more oxygen than the respective PPP lysates supporting the high catalase activity of erythrocytes [18].

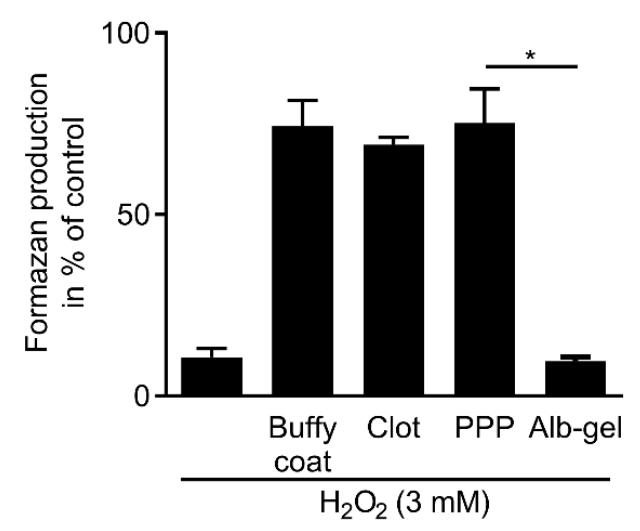

Figure 6. Buffy coat layer and blood clot but not the albumin gel neutralizes $\mathrm{H}_{2} \mathrm{O}_{2}$ cytotoxicity. Gingival fibroblast cells were incubated with a buffy coat layer, blood clot, PPP, and Alb-gel (heated PPP) in the presence of $3 \mathrm{mM} \mathrm{H}_{2} \mathrm{O}_{2}$. MTT results showed that heating at $75^{\circ} \mathrm{C}$ for $10 \mathrm{~min}$ can suppress catalase neutralizing activity while catalase remains active in all the other components. $n=3$. Data represent the mean $\pm \mathrm{SD} .{ }^{*} p<0.05$, by two-tailed Mann-Whitney test. 


\section{Discussion}

Even though PRF is extensively used to support wound healing and bone regeneration, and was recently proposed for facial esthetics [28,29], the underlying molecular and cellular mechanisms remain unclear. The beneficial effects of PRF in alveolar ridge preservation, keratinized tissue augmentation [3], and healing of chronic ulcers [3] might be attributed to its inhibitory effect on osteoclastogenesis [12] along with its pro-angiogenic [30], pro-migratory, proliferative [20], and anti-inflammatory effect [8,11]. Based on the data presented here, PRF might also fight tissue damage and support tissue regeneration by serving as a source of catalase, the evolutionary highly conserved and still mysterious enzyme effectively neutralizing reactive hydrogen peroxide [31]. The main finding of this report is that it is particular the buffy coat and the adjacent red blood layer that showed the strongest catalase activity compared to PPP. Moreover, preparing albumin gels by heating PPP greatly abolishes the catalase activity.

Our findings are in agreement with previous studies showing that activated platelets can efficiently neutralize toxic amounts of hydrogen peroxide by the release of catalase [17]. Consistent with previous reports, the inhibition of the catalase activity by using aminotriazole hindered PRF lysate to exert its protective effect on cells against a high concentration of hydrogen peroxide [17]. The same is true for erythrocytes being a potent source of catalase [18]. The results presented here are also aligned with previous research indicating that hydrogen peroxide at toxic concentrations provokes necrosis and not apoptosis [17]. Moreover, it can be ruled out that the neutralization of the acute toxicity of hydrogen peroxide is not caused by the catalase produced by the gingival fibroblasts. In support of the general understanding of catalase is also the heat sensitivity and that pasteurization of platelet products may decrease or even abolish its activity [21]. Taken together, these findings indicate that PRF, similar to activated platelets, can neutralize hydrogen peroxide-induced necrosis by the release of catalase.

The clinical relevance of the in vitro observations leaves room for speculations. Even though it remains unclear whether platelets are the exclusive source of catalase, these findings provide new perspectives to understand how PRF exerts its beneficial effects in vivo [5]. High amounts of native catalase are present in the healthy skin organ [32] but not in situations where wound healing is impaired by the lack of catalase including skin diseases, such as vitiligo [33], psoriasis [34], and presumably also in refractory leg ulcers [6]. PRF and its respective membrane, and particular the buffy coat layer including parts of the erythrocyte fraction could become a rich source of catalase to support wound healing and may replace or complement traditional clinical strategies where catalase and mimetics are applied for the treatment of skin diseases and wound injury [33,35]. Care should be taken not to pasteurize PRF in the context of virus inactivation and possible allogeneic transplantation, as catalase is temperature-sensitive. Therefore, the clinical application of PRF might be particularly effective at sites of chronic inflammation with low levels of catalase where excessive reactive oxygen species and hydrogen peroxide are not neutralized thereby impairing wound healing.

Considering that chronic inflammatory diseases such as periodontitis are characterized by oxidative stress signals [36] and various reactive oxygen species are liberated under inflammatory conditions [37], PRF might not only exert its anti-inflammatory effect via macrophage polarization [11] but also through neutralization of the hydrogen peroxide-induced inflammation [38]. Catalase might be involved in explaining the positive effects of PRF in refractory skin ulcers [6]. For example, the local application of catalase on thermal skin injury helped to reduce the lesion size in rats [39]. The present data are also relevant to consider heating when preparing an injectable PRF mixture consisting of autologous albumin gel and liquid platelet-rich fibrin. Heating the platelet-poor plasma layer at $75{ }^{\circ} \mathrm{C}$ for 10 min not only changes the resorption properties but also abolishes catalase as well as the glutathione peroxidase activity [24]. It is thus the buffy coat layer keeping the catalase activity of Alb-PRF [24]. A number of limitations should be considered when interpreting the results of this study. Firstly, the origin of the catalase remains unclear but considering that platelets $[16,17]$ and not neutrophils are a major source of catalase [40], the cellular origin can be proposed. Moreover, the number of cells other than platelets in PRF produced at $400 \mathrm{~g}$ is rather low [41]. Second, neutrophils release other reactive 
oxygen species than hydrogen peroxide that may damage cells during overshooting inflammation. Our findings further support the role of the red clot being a rich source of catalase, likely because of the erythrocytes [18]. Future research should also investigate the neutralizing capacity of PRF lysate on reactive oxygen species such as superoxide, hydroxyl radical, singlet oxygen, and alpha-oxygen. Third, platelets also release other detoxifying enzymes that are embedded in the fibrin-rich matrix, therefore, PRF research should not be restricted to the catalase activity. Forth, platelet-rich plasma is also applied clinically covering some of the same indications as PRF, and presumably also contains catalase. This, however, requires to be confirmed experimentally. Finally, it remains unclear whether the catalase can explain some of the clinical outcomes of PRF, not only in the field of dentistry but also with respect particularly to the healing of ulcers.

\section{Conclusions}

Taken together, these results suggest that PRF can neutralize cytotoxic amounts of hydrogen peroxide through the release of catalase. Heating, however, can diminish the catalase activity in PRF.

Supplementary Materials: The following are available online at http://www.mdpi.com/2076-3921/9/6/560/s1, Figure S1: Catalase activity measured by MTT assay, Figure S2: Visualizing bubble assay for catalase activity was analyzed based on the height of oxygen-forming foam, Figure S3: Microscopic illustration for bubbles confirmed that heating inactivates catalase, Table S1: Quantitative activity of catalase for the standard and the samples, Video S1: Microscopic illustration for bubbles by catalase.

Author Contributions: Z.K. contributed to conception and design, contributed to acquisition, analysis, and interpretation; critically revised manuscript; gave final approval; agreed to be accountable for all aspects of work. J.N. contributed to acquisition, analysis, and interpretation; critically revised manuscript; gave final approval; agreed to be accountable for all aspects of work. F.D.S. contributed to acquisition, analysis, and interpretation; critically revised manuscript; gave final approval; agreed to be accountable for all aspects of work. L.P. contributed to acquisition, analysis, and interpretation; critically revised manuscript; gave final approval; agreed to be accountable for all aspects of work. R.J.M. critically revised manuscript; gave final approval; agreed to be accountable for all aspects of work. R.G. contributed to conception and design; contributed to acquisition, analysis, and interpretation; drafted manuscript; critically revised manuscript; gave final approval; agreed to be accountable for all aspects of work. All authors have read and agreed to the published version of the manuscript.

Funding: This research was funded in part by a grant from the Osteology Foundation, Switzerland (17-125 and 17-219). Z.K. and J.N. received support from the Austrian Science Fund (FWF) (4072-B28).

Acknowledgments: The support of Franz J. Strauss for proofreading the manuscript is acknowledged. Open Access Funding by the Austrian Science Fund (FWF).

Conflicts of Interest: Richard Miron declares that he has intellectual property on the Bio-Heat technology utilized in this manuscript. All other authors declare no conflict of interest.

\section{References}

1. Miron, R.J.; Zucchelli, G.; Pikos, M.A.; Salama, M.; Lee, S.; Guillemette, V.; Fujioka-Kobayashi, M.; Bishara, M.; Zhang, Y.; Wang, H.L.; et al. Use of platelet-rich fibrin in regenerative dentistry: A systematic review. Clin. Oral. Investig. 2017, 21, 1913-1927. [CrossRef] [PubMed]

2. Temmerman, A.; Vandessel, J.; Castro, A.; Jacobs, R.; Teughels, W.; Pinto, N.; Quirynen, M. The use of leucocyte and platelet-rich fibrin in socket management and ridge preservation: A split-mouth, randomized, controlled clinical trial. J. Clin. Periodontol. 2016, 43, 990-999. [CrossRef] [PubMed]

3. Temmerman, A.; Cleeren, G.J.; Castro, A.B.; Teughels, W.; Quirynen, M. L-PRF for increasing the width of keratinized mucosa around implants: A split-mouth, randomized, controlled pilot clinical trial. J. Periodontal. Res. 2018, 53, 793-800. [CrossRef] [PubMed]

4. Hamzacebi, B.; Oduncuoglu, B.; Alaaddinoglu, E.E. Treatment of Peri-implant Bone Defects with Platelet-Rich Fibrin. Int. J. Periodontics Restor. Dent. 2015, 35, 415-422. [CrossRef]

5. Strauss, F.J.; Stahli, A.; Gruber, R. The use of platelet-rich fibrin to enhance the outcomes of implant therapy: A systematic review. Clin. Oral. Implant. Res. 2018, 29 (Suppl. 18), 6-19. [CrossRef]

6. Pinto, N.R.; Ubilla, M.; Zamora, Y.; Del Rio, V.; Dohan Ehrenfest, D.M.; Quirynen, M. Leucocyte- and platelet-rich fibrin (L-PRF) as a regenerative medicine strategy for the treatment of refractory leg ulcers: A prospective cohort study. Platelets 2018, 29, 468-475. [CrossRef] 
7. Andreone, A.; den Hollander, D. A Retrospective Study on the Use of Dermis Micrografts in Platelet-Rich Fibrin for the Resurfacing of Massive and Chronic Full-Thickness Burns. Stem. Cells Int. 2019, 2019, 8636079. [CrossRef]

8. Strauss, F.J.; Nasirzade, J.; Kargarpoor, Z.; Stahli, A.; Gruber, R. Effect of platelet-rich fibrin on cell proliferation, migration, differentiation, inflammation, and osteoclastogenesis: A systematic review of in vitro studies. Clin. Oral. Investig. 2020, 24, 569-584. [CrossRef]

9. Kobayashi, E.; Fluckiger, L.; Fujioka-Kobayashi, M.; Sawada, K.; Sculean, A.; Schaller, B.; Miron, R.J. Comparative release of growth factors from PRP, PRF, and advanced-PRF. Clin. Oral. Investig. 2016, 20, 2353-2360. [CrossRef]

10. Castro, A.B.; Herrero, E.R.; Slomka, V.; Pinto, N.; Teughels, W.; Quirynen, M. Antimicrobial capacity of Leucocyte-and Platelet Rich Fibrin against periodontal pathogens. Sci. Rep. 2019, 9, 8188. [CrossRef]

11. Nasirzade, J.; Kargarpour, Z.; Hasannia, S.; Strauss, F.J.; Gruber, R. Platelet-rich fibrin elicits an anti-inflammatory response in macrophages in vitro. J. Periodontol. 2020, 91, 244-252. [CrossRef] [PubMed]

12. Kargarpour, Z.; Nasirzade, J.; Strauss, F.J.; Di Summa, F.; Hasannia, S.; Muller, H.D.; Gruber, R. Platelet-rich fibrin suppresses in vitro osteoclastogenesis. J. Periodontol. 2019, 91, 413-421. [CrossRef] [PubMed]

13. Fruehauf, J.P.; Meyskens, F.L., Jr. Reactive oxygen species: A breath of life or death? Clin. Cancer. Res. 2007, 13, 789-794. [CrossRef] [PubMed]

14. Forman, H.J.; Torres, M. Reactive oxygen species and cell signaling: Respiratory burst in macrophage signaling. Am. J. Respir. Crit. Care Med. 2002, 166 (12 Pt 2), S4-S8. [CrossRef]

15. Doni, M.G.; Avventi, G.L.; Bonadiman, L.; Bonaccorso, G. Glutathione peroxidase, selenium, and prostaglandin synthesis in platelets. Am. J. Physiol. 1981, 240, H800-H803. [CrossRef]

16. Pandey, N.R.; Kaur, G.; Chandra, M.; Sanwal, G.G.; Misra, M.K. Enzymatic oxidant and antioxidants of human blood platelets in unstable angina and myocardial infarction. Int. J. Cardiol. 2000, 76, 33-38. [CrossRef]

17. Kandler, B.; Maitz, P.; Fischer, M.B.; Watzek, G.; Gruber, R. Platelets can neutralize hydrogen peroxide in an acute toxicity model with cells involved in granulation tissue formation. Bone 2005, 36, 671-677. [CrossRef]

18. Agar, N.S.; Sadrzadeh, S.M.; Hallaway, P.E.; Eaton, J.W. Erythrocyte catalase. A somatic oxidant defense? J. Clin. Investig. 1986, 77, 319-321. [CrossRef]

19. Dallegri, F.; Ballestrero, A.; Ottonello, L.; Patrone, F. Platelets as inhibitory cells in neutrophil-mediated cytolysis. J. Lab. Clin. Med. 1989, 114, 502-509.

20. Gruber, R.; Karreth, F.; Kandler, B.; Fuerst, G.; Rot, A.; Fischer, M.B.; Watzek, G. Platelet-released supernatants increase migration and proliferation, and decrease osteogenic differentiation of bone marrow-derived mesenchymal progenitor cells under in vitro conditions. Platelets 2004, 15, 29-35. [CrossRef]

21. Guild, W.R.; Van Tubergen, R.P. Heat Inactivation of Catalase in Deuterium Oxide. Science 1957, 125, 939. [CrossRef] [PubMed]

22. Jung, S.Y.; Kim, H.Y.; Oh, H.J.; Choi, E.; Cho, M.S.; Kim, H.S. Feasibility of autologous plasma gel for tonsil-derived stem cell therapeutics in hypoparathyroidism. Sci. Rep. 2018, 8, 11896. [CrossRef] [PubMed]

23. Doghaim, N.N.; El-Tatawy, R.A.; Neinaa, Y.M.E. Assessment of the efficacy and safety of platelet poor plasma gel as autologous dermal filler for facial rejuvenation. J. Cosmet. Dermatol. 2019. [CrossRef] [PubMed]

24. Fujioka-Kobayashi, M.; Schaller, B.; Mourao, C.; Zhang, Y.; Sculean, A.; Miron, R.J. Biological characterization of an injectable platelet-rich fibrin mixture consisting of autologous albumin gel and liquid platelet-rich fibrin (Alb-PRF). Platelets 2020, 1-8. [CrossRef]

25. Fujioka-Kobayashi, M.; Miron, R.J.; Hernandez, M.; Kandalam, U.; Zhang, Y.; Choukroun, J. Optimized Platelet-Rich Fibrin With the Low-Speed Concept: Growth Factor Release, Biocompatibility, and Cellular Response. J. Periodontol. 2017, 88, 112-121. [CrossRef] [PubMed]

26. Iwase, T.; Tajima, A.; Sugimoto, S.; Okuda, K.; Hironaka, I.; Kamata, Y.; Takada, K.; Mizunoe, Y. A simple assay for measuring catalase activity: A visual approach. Sci. Rep. 2013, 3, 3081. [CrossRef]

27. Andrews, G.P.; Martin, S.E. Heat inactivation of catalase from Staphylococcus aureus MF-31. Appl. Environ. Microbiol. 1979, 37, 1180-1185. [CrossRef]

28. Gentile, R.D. Easy Platelet-Rich Fibrin (Injectable/Topical) for Post-resurfacing and Microneedle Therapy. Facial. Plast. Surg. Clin. North. Am. 2020, 28, 127-134. [CrossRef]

29. Davies, C.; Miron, R.J. PRF in Facial Esthetics; Quintessence Publishing Co Inc.: Batavia, IL, USA, 2020. 
30. De Angelis, P.; De Angelis, S.; Passarelli, P.C.; Liguori, M.G.; Manicone, P.F.; D'Addona, A. Hard and Soft Tissue Evaluation of Different Socket Preservation Procedures Using Leukocyte and Platelet-Rich Fibrin: A Retrospective Clinical and Volumetric Analysis. J. Oral. Maxillofac. Surg. 2019, 77, 1807-1815. [CrossRef]

31. Sepasi Tehrani, H.; Moosavi-Movahedi, A.A. Catalase and its mysteries. Prog. Biophys. Mol. Biol. 2018, 140, 5-12. [CrossRef]

32. Hernandez, A.R.; Boutonnet, M.; Svensson, B.; Butler, E.; Lood, R.; Blom, K.; Vallejo, B.; Anderson, C.; Engblom, J.; Ruzgas, T.; et al. New concepts for transdermal delivery of oxygen based on catalase biochemical reactions studied by oxygen electrode amperometry. J. Control. Release 2019, 306, 121-129. [CrossRef] [PubMed]

33. Schallreuter, K.U.; Moore, J.; Wood, J.M.; Beazley, W.D.; Gaze, D.C.; Tobin, D.J.; Marshall, H.S.; Panske, A.; Panzig, E.; Hibberts, N.A. In vivo and in vitro evidence for hydrogen peroxide (H2O2) accumulation in the epidermis of patients with vitiligo and its successful removal by a UVB-activated pseudocatalase. J. Investig. Dermatol. Symp. Proc. 1999, 4, 91-96. [CrossRef] [PubMed]

34. Zhang, Y.; Li, Z.; Ma, Y.; Mu, Z. Association of total oxidant status, total antioxidant status, and malondialdehyde and catalase levels with psoriasis: A systematic review and meta-analysis. Clin. Rheumatol. 2019, 38, 2659-2671. [CrossRef] [PubMed]

35. Doctrow, S.R.; Lopez, A.; Schock, A.M.; Duncan, N.E.; Jourdan, M.M.; Olasz, E.B.; Moulder, J.E.; Fish, B.L.; Mader, M.; Lazar, J.; et al. A synthetic superoxide dismutase/catalase mimetic EUK-207 mitigates radiation dermatitis and promotes wound healing in irradiated rat skin. J. Investig. Dermatol. 2013, 133, 1088-1096. [CrossRef]

36. Kantarci, A.; Oyaizu, K.; Van Dyke, T.E. Neutrophil-mediated tissue injury in periodontal disease pathogenesis: Findings from localized aggressive periodontitis. J. Periodontol. 2003, 74, 66-75. [CrossRef]

37. Chapple, I.L. Reactive oxygen species and antioxidants in inflammatory diseases. J. Clin. Periodontol. 1997, 24, 287-296. [CrossRef]

38. Ding, Y.; Kim, J.K.; Kim, S.I.; Na, H.J.; Jun, S.Y.; Lee, S.J.; Choi, M.E. TGF-\{beta\}1 protects against mesangial cell apoptosis via induction of autophagy. J. Biol. Chem. 2010, 285, 37909-37919. [CrossRef]

39. Abdel-Mageed, H.M.; Fahmy, A.S.; Shaker, D.S.; Mohamed, S.A. Development of novel delivery system for nanoencapsulation of catalase: Formulation, characterization, and in vivo evaluation using oxidative skin injury model. Artif. Cells Nanomed. Biotechnol. 2018, 46 (Suppl. 1), 362-371. [CrossRef]

40. Roos, D.; Weening, R.S.; Wyss, S.R.; Aebi, H.E. Protection of human neutrophils by endogenous catalase: Studies with cells from catalase-deficient individuals. J. Clin. Investig. 1980, 65, 1515-1522. [CrossRef]

41. Miron, R.J.; Chai, J.; Zheng, S.; Feng, M.; Sculean, A.; Zhang, Y. A novel method for evaluating and quantifying cell types in platelet rich fibrin and an introduction to horizontal centrifugation. J. Biomed. Mater. Res. A 2019, 107, 2257-2271. [CrossRef]

(C) 2020 by the authors. Licensee MDPI, Basel, Switzerland. This article is an open access article distributed under the terms and conditions of the Creative Commons Attribution (CC BY) license (http://creativecommons.org/licenses/by/4.0/). 